\title{
PAINLEVE ANALYSIS OF A CLASS OF NONLINEAR DIFFUSION EQUATIONS
}

\author{
P. CHANDRASEKARAN and E.K. RAMASAMI \\ Bharathiar University \\ Department of Mathematics \\ Coimbatore 641046, INDIA
}

(Received October, 1994; Revised October, 1995)

\begin{abstract}
We study the Painleve analysis for a class of nonlinear diffusion equations. We find that in some cases it has only the conditional Painleve property and in other cases, just the Painleve property. We also obtained special solutions.

Key words: Nonlinear-Diffusion Equation, Painleve Analysis, Painleve Equation, Special Solutions.
\end{abstract}

AMS (MOS) subject classifications: 35Q51.

\section{Introduction}

In recent years, much attention has been focused on higher order nonlinear partial differential equations, known as evolution equations. Such nonlinear equations often occur in the description of chemical and biological phenomena. Their analytical study has been drawing immense interest. A fundamental question when dealing with nonlinear differential equations is "how can one tell beforehand whether or not they are integrable?" Originally, Ablowitz et al [1] conjectured that a nonlinear partial differential equation is integrable if all its exact reductions to ordinary differential equations have the Painleve property: that is, to have no movable singularities other than poles. This approach poses an obvious operational difficulty in finding all exact reductions. This difficulty was circumvented by Weiss et al [10] by postulating that a partial differential equation has the Painleve property if its solutions are single-valued about a movable singular manifold

$$
\phi\left(z_{1}, z_{2}, \ldots, z_{n}\right)=0
$$

where $\phi$ is an arbitrary function. In other words, a solution $u\left(z_{i}\right)$ of a partial differential equation should have a Laurent-like expansion about the movable singular manifold $\phi=0$ :

$$
u\left(z_{i}\right)=\left[\phi\left(z_{i}\right)\right]^{\alpha} \sum_{j=0}^{\infty} u_{j}\left(z_{i}\right) \phi\left(z_{i}\right)^{j}
$$

where $\alpha$ is a negative integer. The number of arbitrary functions in expansion (1.2) should be equal to the order of the partial differential equation. Inserting expansion (1.2) into the targeted equation yields a recurrence formula that determines $u_{n}\left(z_{i}\right)$ for all $n>0$, except for a finite number of $r_{1}, r_{2}, r_{3}, \ldots, r_{j}>0$, called resonances. For some equations, the recurrence formulas at the resonance values may result in constraint equations for the movable singular manifold which implies that it is no longer completely arbitrary. In such cases, one can say that the equation has 
the Conditional Painleve property [8]. The Painleve property is a sufficient condition for the integrability or solvability of equations. Meanwhile, various authors have applied this approach to other nonlinear partial differential equations to decide whether or not these equations are integrable. Recent investigations of Cariello and Tabor [3] regarding the Painleve analysis also yield a systematic procedure for obtaining special solutions when an equation possesses only the conditional Painleve property. Fisher [4] proposed the nonlinear diffusion equation

$$
u_{t}=D u_{x x}+\beta u(1-u)
$$

as a model for the propagation of a mutant gene with an advantageous selection of intensity $\beta$. Roy Choudhury [8] has considered the extended form of equation (1.3) as

$$
u_{t}=\beta u^{p}\left(1-u^{q}\right)+D\left(u^{m} u_{x}\right)_{x}
$$

for Painleve analysis and obtained special solutions for various cases of $p, q$, and $m$.

In this paper we consider

$$
u_{t}=\beta u^{p}\left(1-u^{q}\right)+\mu u^{s} u_{x}+D\left(u^{m} u_{x}\right)_{x}
$$

which is a generalization of (1.4) for the Painleve analysis. This equation has several interesting limiting cases which have already been studied:

(i) when $\mu=m=0, p=1$, and $q \neq 0$, equation (1.5) is reduced to the generalized Fisher equation. For $q=1$, equation (1.5) reduces to the Fisher equation and for $q=2,(1.5)$ reduces to the Newell Whitehead equation.

(ii) If we take $\beta=m=0$, then equation (1.5) is reduced to the generalized Burgers' equation. With $s=1$ and $\beta=m=0$, equation (1.5) gives the Burgers' equation, which describes the far field of wave propagation in nonlinear dissipative systems [11].

(iii) When $m=0, p=1$, and $q=s$, equation (1.5) is reduced to the generalized BurgerFisher equation [9].

\section{Painleve Analysis of Equation (1.5)}

\subsection{Leading order and resonance analyses}

The behavior of solutions of equation (1.5) at a movable singular manifold [7],

$$
\phi(x, t)=0
$$

is determined by a leading order analysis whereby one makes the substitution

$$
u(x, t)=u_{0}(x, t)[\phi(x, t)]^{\alpha}
$$

and balances the most singular or dominant terms. Substituting (2.2) into (1.5), we obtain three possible values for $\alpha$ as follows: obtain

Case (i): $\quad p+q>m \geq s: \quad$ Balancing the dominant terms $u^{p+q}, m u^{m-1} u_{x}^{2}$, and $u^{m} u_{x x}$, we

$$
\alpha=-2 /(p+q-m-1)
$$

and 


$$
\beta u_{0}^{p+q-m-1}=2 D(p+q+m+1) \phi_{x}^{2} /(p+q-m-1)^{2} .
$$

Case (ii): $p+q>s>m=0$ : Balancing the dominant terms $u^{p+q}$ and $u^{s} u_{x}$, we obtain

$$
\alpha=-1 / p+q-s-1
$$

and

$$
\beta u_{0}^{p+q-s-1}=(-\mu / p+q-s-1) \phi_{x}
$$

For $p+q=-m+2 s+1>s>m$,

$$
\alpha=-1 /(s-m)
$$

Here we have two branches for $u_{0}$ as follows:

$\operatorname{branch}(i): u_{0}=-(k+1) \phi_{x} ;$ and branch $(i i): u_{0}=k \phi_{x}$, where $k=1,2,3, \ldots \ldots \ldots$,

$$
m=\left((2 k+1)^{2}-9\right) / 4, \text { and } \beta=\mu=D=1 .
$$

Case (iii): $s>m \geq p+q$ : Balancing the dominant terms $u^{s} u_{x}, m u^{m-1} u_{x}^{2}$, and $u^{m} u_{x x}$, we obtain

$$
\alpha=-1 /(s-m)
$$

and

$$
u_{0}^{s-m}=(D / \mu)((1+s) /(s-m)) \phi_{x}
$$

We have the following lemma as a result.

Lemma: For all combinations of integer values of $p, q, m$ and $s$, the leading order singularity of equation (1.5) is

(i) a movable pole for all combinations with $(p+q-m-1)$ is equal to 1 or 2 for case $(i)$, with $(p+q-s-1)$ being equal to 1 for case (ii), and with $s-m$ being equal to 1 for case (iii);

(ii) a rational branch point for all combinations with $(p+q-m-1)>2$ for case $(i)$, $(p+q-s-1)>1$ for case (ii), and $s-m>1$ for case (iii).

The powers of $\phi$, at which the arbitrary coefficient appears in the series, that is, the resonances, are determined by setting

$$
u(x, t)=u_{0}(x, t)(\phi(x, t))^{\alpha}+p(\phi(x, t))^{\alpha+r}
$$

and balancing the most singular terms of equation (1.5) again. We obtain for case $(i)$, using the value of $\alpha$ given by (2.3),

$$
\begin{gathered}
p\left\{(r+\alpha)^{2}+(2 m \alpha-1)(r+\alpha)+\left[m \alpha(\alpha-1)+m(m-1) \alpha^{2}\right.\right. \\
\left.\left.-2(p+q)(p+q+m+1) /(p+q-m-1)^{2}\right]\right\}=0
\end{gathered}
$$

with solutions

$$
r=-1,2[1-\alpha(m+1)]
$$


However, for case (ii), with value $\alpha$ given in (2.7) and for a particular value of $m$ given by (2.8), we obtain for branch $(i)$

$$
\begin{gathered}
p\left\{\left(2 m \alpha(r+\alpha)+\alpha^{2} m(m-1)+(r+\alpha)(r+\alpha-1)+\alpha(\alpha-1) m\right)\right. \\
\left.-(s \alpha+(r+\alpha))(k+1)-(-m+2 s+1)(k+1)^{2}\right\}=0
\end{gathered}
$$

with solutions

$$
r=-1,\{(k+3)-2 \alpha(m+1)\}
$$

and for branch (ii)

$$
\begin{gathered}
p\left\{\left(2 m \alpha(r+\alpha)+\alpha^{2} m(m-1)+(r+\alpha)(r+\alpha-1)+\alpha(\alpha-1) m\right)+(s \alpha+(r+\alpha)) k\right. \\
\left.+(-m+2 s+1) k^{2}\right\}=0
\end{gathered}
$$

with solutions

$$
r=-1 \text { and } r=(2-k)-2 \alpha(m+1) .
$$

For case (iii), we get

$$
p\left\{r^{2}+r(2 \alpha(m+1))-r+\alpha^{2}(1+m)^{2}-\alpha(1+m)+\alpha s(r+\alpha) u_{0}^{s-m}\right\}=0
$$

with solutions

$$
r=-1 \text { and } r=-\alpha(1+s)
$$

Hence, we have the following theorem.

Theorem: Equation (1.5) does not have the Painleve property for all combinations of $p, q, m$ and $s$ with $((p+q+m+1) /(p+q-m-1)) \leq 0$ for case $(i)$, with $((k+1) s-k m+1) /(s$ $-m) \leq 0, \beta=\mu=D=1$ and $m=\left((2 k+1)^{2}-9 / 4\right), k=1,2, \ldots, m=0,1,2, \ldots$ for case $(i i)$ and with $(s+1) /(s-m) \leq 0$ for case $(i i i)$.

By using the above lemma and theorem, we consider the following cases:

(i) $\quad m=s=0, p=1$ and $q=2$;

(ii) $\quad m=0, s=1, p=1$ and $q=2$;

(iii) $\quad m=0, p=0, q=0$ and $s=1$;

(iv) $\quad m=1, p=0, q=0$ and $s=2$;

(v) $\quad m=2, p=q=1$ and $s=3$,

in which equation (1.5) has a movable pole as leading order singularity, and therefore, it may have a valid Laurent expansion.

\section{Generalized Laurent Expansions}

\subsection{Equation (1.5) with $p=1, m=s=0, q=2$}

In this case, we consider equation 


$$
u_{t}=\beta\left(u-u^{3}\right)+\mu u_{x}+D u_{x x} .
$$

The generalized Laurent expansion (with $\alpha=-1$ ) takes the form

$$
u=u_{0} \phi^{-1}+u_{1}+u_{2} \phi+u_{3} \phi^{2}+u_{4} \phi^{3}
$$

where $r=-1,4$ and $u_{4}$ should be an arbitrary function.

Now substituting (3.2) into (3.1) and using the Kruskal substitution [6] we have that

$$
\phi(x, t)=x-\sigma(t), \text { and } u_{j}(x, t)=u_{j}(t) .
$$

Here $\sigma$ is an arbitrary function. Using (3.2) and (3.3) in (3.1) and collecting coefficients of equal powers of $\phi$, we have:

$$
\begin{gathered}
\phi^{-3}: u_{0}=\sqrt{(2 D / \beta)} \\
\phi^{-2}: u_{1}=(-1 / 6 D) \sqrt{(2 D / \beta)}\left(\mu+\sigma_{t}\right) \\
\phi^{-1}: u_{2}=\left(-1 /(6 D)^{2}\right) \sqrt{(2 D / \beta)}\left(\mu+\sigma_{t}\right)^{2}+(1 / 3) \sqrt{(\beta / 2 D)} \\
\phi^{0}: u_{3}=(-1 /(4 D)) \sqrt{(2 D / \beta)}\left[\left(-\sigma_{t t} / 6 D\right)+16\left(\mu+\sigma_{t}\right)^{3} / 216 D^{2}\right]+\sqrt{(\beta / 2 D)}\left(\mu+\sigma_{t}\right) / 6 D \\
\phi^{1}: 0 X u_{4}=\left(8 /(6 D)^{2}\right) \sqrt{(2 D / \beta)}\left(\mu+\sigma_{t}\right) \sigma_{t t} \\
-\left[16 \sqrt{(2 D / \beta)}\left(\mu+\sigma_{t}\right)^{4} /(6 D)^{3}\right]+4(\sqrt{(\beta / 2 D)})\left(\mu+\sigma_{t}\right)^{2} / 6 D,
\end{gathered}
$$

where $\sigma_{t}=d \sigma / d t$, and $\sigma_{t t}=d^{2} \sigma / d t^{2}$. From (3.8), $u_{4}$ is an arbitrary function of this order if and only if the right-hand side of (3.8) is zero: that is, if and only if the singularity manifold $\phi$ is subject to a certain constraint. Therefore, system (3.1) does not have the full Painleve property, but only the Conditional Painleve property.

\subsection{Equation (1.5) with $p=1, m=0, q=2, s=1$}

In this case, we consider equation

$$
u_{t}=\beta\left(u-u^{3}\right)+\mu u u_{x}+D u_{x x} .
$$

This is the generalized Burgers-Fisher equation with cubic nonlinearity. form

Let us assume that $\beta=\mu=D=1$. Let us take the generalized Laurent expansion of the

$$
u=u_{0} \phi^{-1}+u_{1}+u_{2} \phi+u_{3} \phi^{2}
$$

corresponding to the resonances at $r=-1,3$ obtained from (2.12) for branch $(i i)$.

Substituting (3.10) into (3.9) and collecting coefficients of equal powers of $\phi$, we have:

$$
\begin{gathered}
\phi^{-3}: \quad u_{0}=1,2 \\
\phi^{-2}: \quad u_{1}=-\sigma_{t} / 4 \\
\phi^{-1}: \quad u_{2}=\left(\sigma_{t}^{2} / 16\right)+(1 / 3)
\end{gathered}
$$




$$
\phi^{0}: 0 X u_{3}=\left(-\sigma_{t}^{3} / 8\right)+\left(\sigma_{t t} / 4\right)+\left(\sigma_{t} / 2\right)
$$

Again, we find that $u_{3}$ is arbitrary only if $\phi$ satisfies a certain constraint. Hence, system (3.9) possesses the Conditional Painleve property.

\subsection{Equation (1.5) with $p=q=m=0, s=1$}

In this case, equation (1.5) reduces to

$$
u_{t}=\mu u u_{x}+D u_{x x}
$$

This equation is the well-known Burgers' equation whose Painleve property is studied by Weiss et al [10].

\subsection{Equation (1.5) with $p=q=0, m=1, s=2$}

In this case, equation (1.5) becomes

$$
u_{t}=\mu u^{2} u_{x}+D u u_{x x}+D u_{x}^{2}
$$

Using (2.9) and (2.13), we obtain

$$
u_{0}=(3 D / \mu) \phi_{x}
$$

and the resonances are $r=-1$ and 3. Hence, we take the Laurent expansion of the form

$$
u=u_{0} \phi^{-1}+u_{1}+u_{2} \phi+u_{3} \phi^{2} .
$$

Substituting (3.17) into (3.16) and collecting coefficients of equal powers of $\phi$, we have

$$
\begin{gathered}
\phi^{-4}: \quad u_{0}=(3 D / \mu) \\
\phi^{-3}: \quad u_{1}=0 \\
\phi^{-2}: \quad u_{2}=(-1 / 3 D) \sigma_{t} \\
\phi^{-1}: \quad 0 X u_{3}=0 .
\end{gathered}
$$

Equation (3.21) shows that $u_{3}$ is an arbitrary function. Therefore, (3.16) possesses the Painleve property.

3.5 Equation (1.5) with $p=q=1, m=2, s=3$

In this case, equation (1.5) becomes

$$
u_{t}=\beta\left(u-u^{2}\right)+\mu u^{3} u_{x}+D u^{2} u_{x x}+D u u_{x}^{2} .
$$

We take the Laurent expansion of the form

$$
u=u_{0} \phi^{-1}+u_{1}+u_{2} \phi+u_{3} \phi^{2}+u_{4} \phi^{3}
$$

corresponding to the resonances $r=-1$ and 4 .

Substituting (3.23) into (3.22) and collecting coefficients of equal powers of $\phi$, we get: 


$$
\begin{gathered}
\phi^{-5}: u_{0}=(4 D / \mu) \\
\phi^{-4}: u_{1}=0 \\
u^{-3}: \quad u_{2}=0 \\
\phi^{-2}: \quad u_{3}=\left(-\mu^{2} \sigma_{t} / 16 D^{2}\right)-\beta \mu / 4 D \\
\phi^{-1}: 0 X u_{4}=4 D \beta / \mu .
\end{gathered}
$$

The right-hand side of (3.28) shows that $u_{4}$ is arbitrary only if $\beta=0, D \neq 0$. For $D=0, \beta \neq 0$, (3.22) does not have the Painleve property. Therefore, (3.22) possesses the Painleve property only if $\beta=0$.

\subsection{Comparison of the results of equation (1.5) with those of (1.4)}

In studying the Painleve property for nonlinear differential equations, dominant terms of the equation determine the leading order terms, the Laurent expansion and the resonances. For the case $p+q>m \geq s$, the dominant terms of equation (1.5) turn out to be the same as those of (1.4). Therefore, in this case, the results of leading order balance for equation (1.5) agree with those of equation (1.4) and hence, we have the same structure of Laurent expansions and the resonances for equation (1.5) and equation (1.4). For (1.5), with the values of $p, q, m$ and $s$, as above, we find the constraint equations (for example, (3.8) in case $(i)$ and (3.14) in case (ii)) for the movable singularity manifold corresponding to the last resonance value. This shows that the singularity manifold is not arbitrary as in the case of (1.4) in [8] for certain values of $p, q$, and $m$. Therefore, equation (1.5) for case $(i)$ possesses the Conditional Painleve property for certain values of $p, q, m$ and $s$. Other cases of (1.5), having either the Painleve property or the Conditional Painleve property, are not applicable to (1.4).

\subsection{Special solutions}

We are able to find special solutions to some particular cases.

\subsubsection{Equation (1.5) with $p=1, q=2, m=s=0$}

Let $\phi=A+B e^{m z}$, where

$$
z=x-c t
$$

and

$$
u=u_{0} \phi^{-1} \text {. }
$$

Substituting (3.30) into (3.1), we obtain

$$
\begin{gathered}
\phi^{-1}: \quad-\phi_{t}-3 D \phi_{x x}-\mu \phi_{x}=0 \\
\phi^{-2}: \quad D \phi_{x x x}+\mu \phi_{x x}-\phi_{x t}+\beta \phi_{x}=0 .
\end{gathered}
$$

Now substituting (3.29) into (3.31) and (3.32), we find that

$$
m=\sqrt{(\beta / 2 D)} \text { and } c=-3 \sqrt{(\beta D / 2)}-\mu .
$$

Using (3.29), (3.30), and (3.33), we obtain the solution for (3.1) in the form 


$$
u=1 /\left(1+A e^{m z}\right) \text { where } z=x+(3 \sqrt{(\beta D / 2)}+\mu) t .
$$

3.7.2 Equation (1.5) with $p=1, q=2, m=0, s=1$

Substituting (3.30) into (3.9), we obtain

$$
\begin{gathered}
\phi^{-3}: u_{0}=\phi_{x} \text { or } u_{0}=-2 \phi_{x} \\
\phi^{-2}: \phi_{t}-2 \phi_{x x}=0 \\
\phi^{-1}:-\phi_{x t}+\phi_{x x x}+\phi_{x}=0 .
\end{gathered}
$$

Substituting (3.29) into (3.36) and (3.37), we obtain for $u_{0}=\phi_{x} u=1 /\left(1+A e^{-m z}\right)$, where $z=x-2 t, m=1$ and $A$ is an arbitrary constant.

\subsubsection{Reduction of (3.1) to Painleve canonical equation}

Without loss of generality, we may assume that $\beta=\mu=D=1$. Then equation (3.1) becomes

$$
u_{t}=u-u^{3}+u_{x}+u_{x x}
$$

We use the method of Ablowitz and Zeppetella [2] to reduce (3.38) to the Painleve canonical equation of type $I$.

Let

$$
u(z)=f(z) w(s)+g(z), s=h(z), \text { and } z=x-c t .
$$

Substituting (3.39) into (3.38), we find that

$$
\begin{aligned}
w^{\prime \prime}\left(h^{\prime}\right)^{2}= & -w^{\prime} h^{\prime}\left(\left(2 f^{\prime} / f\right)+\left(h^{\prime \prime} / h\right)+(c+1)\right)-\left((1+c)\left(f^{\prime} / f\right)+\left(f^{\prime \prime} / f\right)+1\right) w \\
& +3 g^{2} w+f^{2} w^{3}-(1 / f)\left((1+c) g+g^{\prime \prime}+g-g^{3}\right)+3 f w^{2} g .
\end{aligned}
$$

We take $g=0$ and $f$ and $h$ so that

$$
\begin{gathered}
\left(2 f^{\prime} / f\right)+\left(h^{\prime \prime} / h^{\prime}\right)=-(1+c) \\
(c+1)\left(f^{\prime} / f\right)+\left(f^{\prime \prime} / f\right)+1=0 .
\end{gathered}
$$

Solving (3.41) and (3.42), we have

$$
\begin{gathered}
f(z)=\exp (\delta z), \text { where } \delta=\left[-(c+1) \pm \sqrt{\left((c+1)^{2}-4\right)}\right] / 2 \\
h(z)=\tau \exp (-(c+1)+2 \delta) z .
\end{gathered}
$$

We choose

$$
c= \pm(3 / \sqrt{2})-1, \quad \delta=-1 / \sqrt{2}, \text { and } \tau=1 .
$$

Substituting (3.41) through (3.45) into (3.40), we have 


$$
\left(d^{2} w / d s^{2}\right)=2 w^{3} .
$$

This equation may be regarded as a canonical equation of the type characterized by $L(z, w)=0$ [5]. Furthermore, it is integrable in terms of the Jacobi elliptic functions, whose squared modulus is one-half $\left(k^{2}=\frac{1}{2}\right)$. An elliptic function of this type is called a lemniscate function [12].

\subsubsection{Reduction of (3.16) to Riccati equation}

Let $u=f(z)$, where

$$
z=x-c t
$$

Substituting (3.47) into (3.16) with $\mu=D=1$, we obtain

$$
c f^{\prime}+f^{2} f^{\prime}+f f^{\prime \prime}+f^{\prime 2}=0 .
$$

Integrating (3.48) once, we get

$$
f^{\prime}=-\left(c+\left(f^{2} / 3\right)\right)
$$

Equation (3.49) is a Riccati equation [5], which can be linearized through the transformation

$$
f=3 y^{\prime} / y
$$

Substituting (3.50) into (3.49), we get

$$
y^{\prime \prime}=(-c / 3) y(z)
$$

which is a second order linear differential equation. Solving (3.51), we obtain

$$
y(z)=A \cos \sqrt{(c / 3)} z+B \sin \sqrt{(c / 3)} z
$$

Using (3.52), (3.50) becomes

$$
f(z)=\sqrt{(3 c)}((-A \sin \sqrt{(c / 3)} z+B \cos \sqrt{(c / 3)} z) /(A \cos \sqrt{(c / 3)} z)+B \sin \sqrt{(c / 3)} z=u(x, t) .
$$

\section{Acknowledgements}

The authors are thankful to the referee whose suggestions improved the presentation of the paper.

\section{References}

[1] Ablowitz, M.J., Ramani, A. and Segur, H., A connection between nonlinear evolution equations and ordinary differential equations of P-type I, J. Math. Phys. 21 (1980), 715721.

[2] Ablowitz, M.J. and Zeppetella, A., Explicit solutions of Fisher's equation for a special wave speed, Bull. Math. Bio. 41 (1979), 835-840.

[3] Cariello, F. and Tabor, M., Painleve expansions for nonintegrable evolution equations, Physica D 39 (1989), 77-94. 
[4] Fisher, R.A., The wave of advance of advantageous genes, Ann. Engenics 7 (1937), 355369 .

[5] Ince, E.L., Ordinary Differential Equations, Dover, New York 1956.

[6] Jimbo, M., Kruskal, M.D. and Miwa, T., Painleve test for the self-dual Yang-Mills equation, Phys. Lett. A 92 (1982), 59-60.

[7] Ramani, A., Grammaticos, B. and Bountis, T., The Painleve property and singularity analysis of integrable and nonintegrable systems, Phys. Reports 180 (1989), 159-245.

[8] Roy Choudhury, S., Painleve analysis and partial integrability of a class of reaction-diffusion equations, Nonlinear Analysis: TMA 18 (1992), 445-459.

[9] Wang, X.Y., Zhu, Z.S. and Lu, Y.K., Solitary wave solutions of the generalized BurgersHuxley equation, J. Phys. A: Math. Gen. 23 (1990), 271-274.

[10] Weiss, J. Tabor, M. and Carnevale, G., The Painleve property for partial differential equations, J. Math. Phys. 24 (1983), 522-526.

[11] Whitham, G.B., Linear and Nonlinear Waves, Wiley, New York 1974.

[12] Whittaker, E.T. and Watson, G.N., A Course of Modern Analysis, Cambridge University Press, Cambridge 1927. 


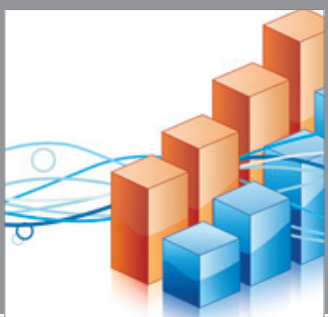

Advances in

Operations Research

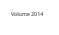

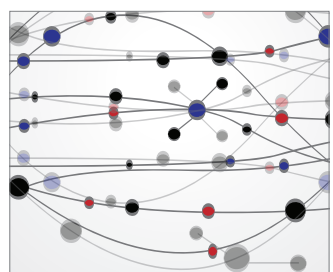

\section{The Scientific} World Journal
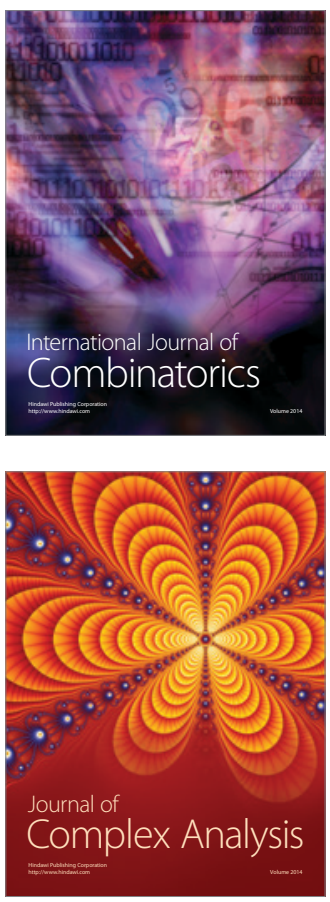

International Journal of

Mathematics and

Mathematical

Sciences
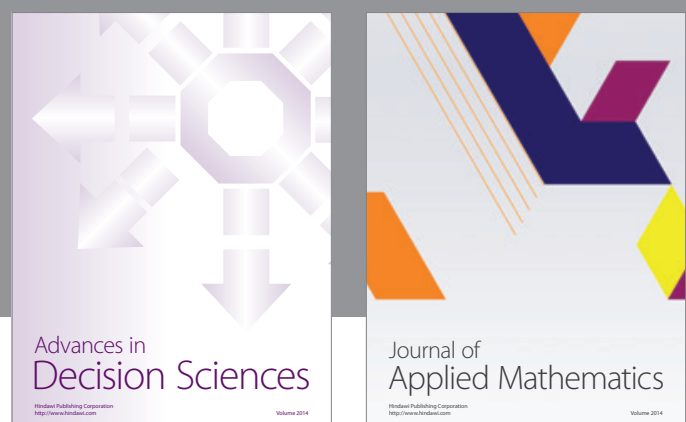

Journal of

Applied Mathematics
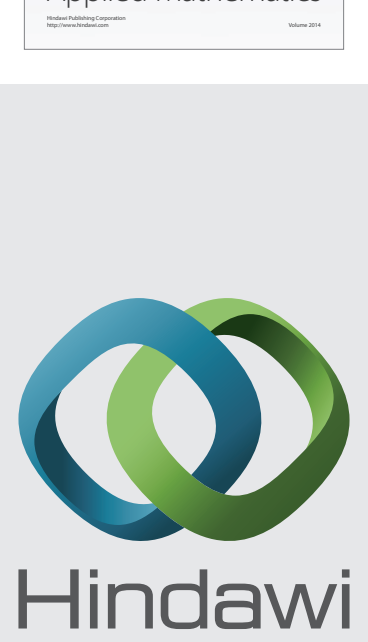

Submit your manuscripts at http://www.hindawi.com
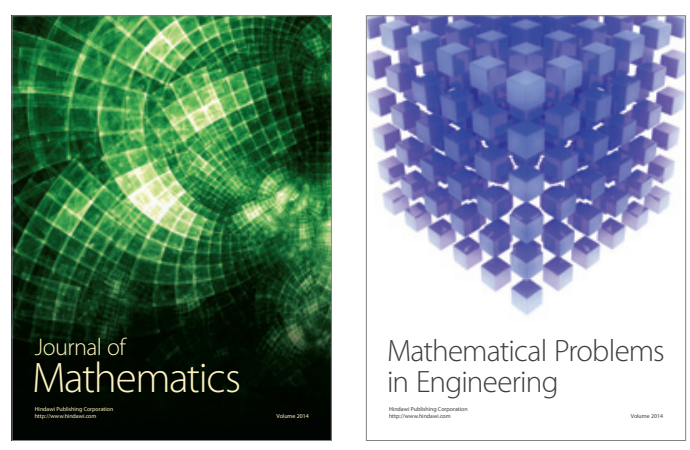

Mathematical Problems in Engineering
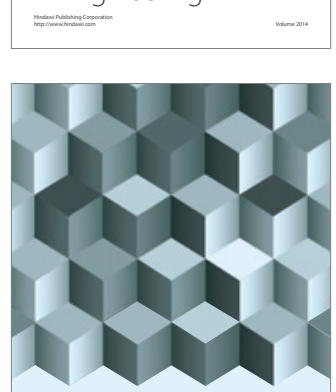

Journal of

Function Spaces
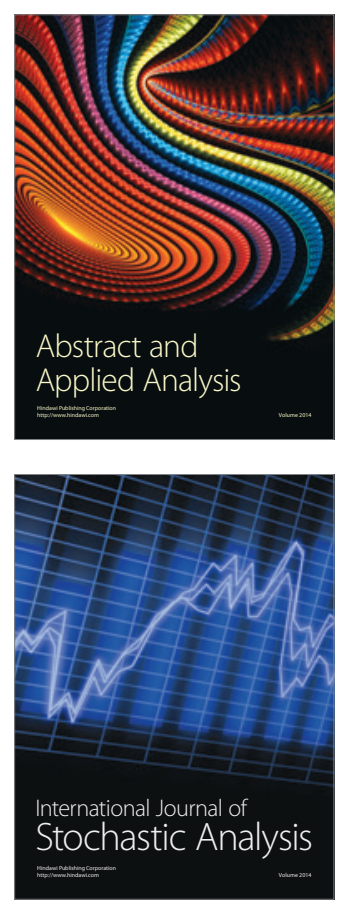

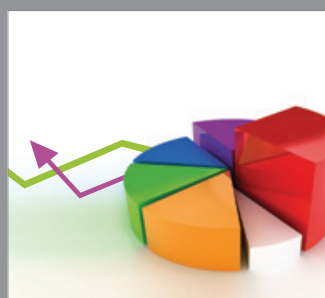

ournal of

Probability and Statistics

Promensencen
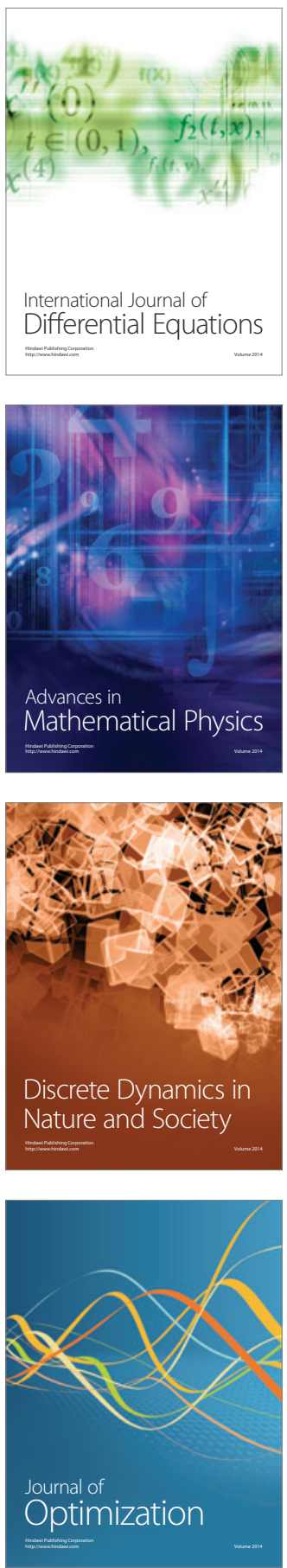